\section{Noonan syndrome associated with growth hormone deficiency with biallelic LZTR1 variants}

We read with great interest the paper by Johnston et al. about Noonan syndrome (NS) associated with LTZR1, the first with biallelic variants. ${ }^{1}$ Here we report a patient with growth hormone $(\mathrm{GH})$ deficiency and Noonan syndrome also with biallelic LTZR1 variants. One of the patients reported by Johnston et al. (proband of family 3, individual II-4) also had GH deficiency. Although short stature is a frequent finding in Noonan syndrome (NS), its pathophysiology remains unknown.

Our patient was born at $3330 \mathrm{~g}$ and $50 \mathrm{~cm}$ to nonconsanguineous parents. Postnatal echocardiography demonstrated transposition of the great vessels, pulmonary stenosis, interventricular and interatrial communication, and he underwent Senning correction surgery at the age of 8 months. $\mathrm{He}$ also had typical facial features of NS including ptosis, triangular face, high-arched palate, low-set ears, and micrognathia, in addition to pectus excavatum.

At the age of 12.5 years, he was diagnosed with GH deficiency. His height was $123.6 \mathrm{~cm}$ (SDS -3.5), insulin-like growth factor 1 concentration $46 \mu \mathrm{g} / \mathrm{L}$ (reference for sex and age 63 to $761 \mu \mathrm{g} / \mathrm{L}$ ) and $\mathrm{GH}$ peak after clonidine and glucagon stimulation tests was only $1.4 \mu \mathrm{g} / \mathrm{L}$ (normal $>5 \mu \mathrm{g} / \mathrm{L}$ ). At the chronological age of 14 years he had a bone age of 7 years. He was treated with human recombinant GH (rhGH, Somatropin) from 12.5 to 22 years reaching a final adult height of $157.5 \mathrm{~cm}$. Retesting during the transition phase confirmed GH deficiency. Pituitary magnetic resonance imaging (MRI) revealed thickening of the left side of the optic chiasm suggestive of glioma and no other abnormalities. There was no characteristics of schwannomatosis.

The patient's DNA was submitted to a targeted gene panel containing 64 genes associated with short stature including 17 genes associated with NS (PTPN11, SOS1, SOS2, NF1, NRAS, HRAS, KRAS, SHOC2, BRAF, RAF1, MAP2K1, MAP2K2, CBL, SPRED1, RASA2, RIT1, LZTR1) and 24 genes for GH deficiency (capture and sequencing with Agilent and Illumina technologies, respectively). The analysis revealed compound heterozygosity for LZTR1 variants and was negative for the other panel genes. The patient has a missense pathogenic variant c.881G>T:p.Arg294Leu NM_006767.3 (gnomAD 0, CADD 36) inherited from his mother and a stop-gain variant c.2212C>T:p.Gln738* (ExAC 0.00001657, gnomAD 0.00001805 , CADD 43) inherited from his father and also present in both of his brothers, all of whom have normal phenotype and height. The missense variant is in the Kelch_5 motif of the epithiospecifier domain and the stop-codon in the BTB domain of LTZR1.

The molecular mechanisms relating LTZR1 alterations to NS or GH deficiency remain to be elucidated. Association of NS and GH deficiency is not frequent but the present patient and one patient reported by Johnston et al. suggest that this occurrence may be more frequent when associated to LZTR1 variants.

\section{FUNDING}

São Paulo Research Foundation (FAPESP) also supported this work: grant number 2013/03236-5 to AALJ; 2015/26563-7 to LRC; grant 2013/02162-8 and grant SELA 2014/50137-5.

\section{DISCLOSURE}

The authors declare no conflicts of interest

\section{Marilena Nakaguma ${ }^{1}$, Alexander A. L. Jorge ${ }^{2}$ and} Ivo J. P. Arnhold ${ }^{1}$

${ }^{1}$ Unidade de Endocrinologia do Desenvolvimento, Laboratório de Hormônios e Genética Molecular LIM42, Hospital das Clínicas, Disciplina de Endocrinologia, Faculdade de Medicina da Universidade de São Paulo, São Paulo, Brazil; ${ }^{2}$ Unidade de Endocrinologia Genética, Laboratório de Endocrinologia Celular e Molecular LIM25, Disciplina de Endocrinologia, Hospital das Clínicas da Faculdade de Medicina da Universidade de São Paulo, São Paulo, Brazil. Correspondence:

Marilena Nakaguma (marilena.nakaguma@fm.usp.br)

\section{REFERENCES}

1. Johnston JJ, van der Smagt JJ, Rosenfeld JA, et al. Autosomal recessive Noonan syndrome associated with biallelic LZTR1 variants. Genet Med. 2018. https://doi.org/10.1038/gim.2017.249.

Advance online publication 30 June 2018. doi:10.1038/s41436-018-0041-5 